Proyecciones

Vol. 18, No 1, pp. 1-11, July 1999

Universidad Católica del Norte

Antofagasta - Chile

\title{
A NEW CONVERGENCE THEOREM FOR THE METHOD OF TANGENT HYPERBOLAS IN BANACH SPACE
}

\author{
IOANNIS K. ARGYROS \\ Cameron University, U.S.A.
}

\begin{abstract}
In this study we approximatc a locally unique solution of a nonlinear operator equation in Banach space using the method of tangent hyperbolas. A neu semilocal convergence theorem is provided using Lipschitz conditions on the second Fréchet-derivative. Our conditions are different than earlier ones. Hence, they have theoretical and practical value. Numerical examples are also provided.
\end{abstract}

AMS (MOS) Subject Classification : 65B05, 47H17, 49D15.

Key Words and Phrases : Banach space, method of tangent hyperbolas, Fréchct-derivative, Ncuton - Kantorovich hypothesis. 


\section{Introduction}

In this study we are concerned with the problem of approximating a locally unique solution $x^{*}$ of the equation

$$
F(x)=0,
$$

where $F$ is a twice Fréchet-differentiable operator defined on a convex subset $D$ of a Banach space $E$ with values in itself.

The method of tangent hyperbolas (Halley)

$$
\begin{array}{r}
x_{n+1}=x_{n}-\left[I-\frac{1}{2} F^{\prime}\left(x_{n}\right)^{-1} F^{\prime \prime}\left(x_{n}\right) F^{\prime}\left(x_{n}\right)^{-1} F\left(x_{n}\right)\right]^{-1} \\
F^{\prime}\left(x_{n}\right)^{-1} F\left(x_{n}\right)\left(x_{0} \in D\right)(n \geq 0)
\end{array}
$$

is a cubically convergent method, usually employed to approximate a solution $x^{*}$. Here $F^{\prime}(x), F^{\prime \prime}(x)(x \in D)$ denote the first and second Fréchetderivatives of the operator $F$ at $x \in D$. Note that $F^{\prime}(x) \in L(E, E)$, the space of bounded linear operators from $E$ into $E$ and $F^{\prime \prime}(x) \in L(E, L(E, E))$.

Sufficient convergent conditions for this method have been given by niany authors [1], [2], [3], [5], [6], [9], [11]. In our study, we use Taylor's theorem for a twice Fréchet-differcntiable operator and weaker Lipschitz conditions on the second Fréchet-derivative. This way we provide sufficicnt conditions different than earlier ones. Hence, our results have theorctical as well as practical value.

Finally, we complete our study by providing a numerical example to show that the method of tangent parabolas starting from $x_{0}$ converges to $x^{*}$, whercas the same cannot be guaranteed by existing methods [1], [2], [3], [5], [6], [9], [11].

\section{Convergence Analysis}

We state a theorem whose proof can be found in $[5],[6],[9],[11]$, for comparison:

Theorem 1. Let $F: D \subseteq E_{1} \rightarrow E_{2}$ be a twice Fréchet-differcntiable operator. Assume:
(a) $F^{\prime}\left(x_{0}\right)^{-1} \in L(E, E)\left(x_{0} \in D\right)$;
(b) $d_{1} \geq\left\|F^{\prime}\left(x_{0}\right)^{-1} F\left(x_{0}\right)\right\|, b_{1} \geq\left\|F^{\prime}\left(x_{0}\right)^{-1} F^{\prime \prime}\left(x_{0}\right)\right\|$; 
(c) $\left\|F^{\prime}\left(x_{0}\right)^{-1}\left[F^{\prime \prime}(x)-F^{\prime \prime}(y)\right]\right\| \leq a_{1}\|x-y\|$ for all $x, y \in D$;

(d) $6 d_{1} b_{1}^{3}+9 d_{1}^{2} a_{1}^{2}+18 d_{1} b_{1} a_{1}-3 b_{1}^{2}-8 a_{1} \leq 0$.

Then,

(i) the scalar polynomial

$$
\delta(t)=\frac{1}{6} a_{1} t^{3}+\frac{1}{2} b_{1} t^{2}-t+d_{1}
$$

has two positive zeros $\gamma_{1}$ and $\gamma_{2}$ with $\gamma_{1} \leq \gamma_{2}$, and a negative zero $\gamma_{3}$;

(ii) the method of tangent hyperbolas is well defined, remains in $\bar{U}\left(x_{0}, \gamma_{1}\right)=$ $\left\{x \in E \mid\left\|x-x_{0}\right\| \leq \gamma_{1}\right\}$ for all $n \geq 0$, and converges to a unique solution $x^{*}$ of equation $F(x)=0$ in $U\left(x_{0}, \gamma_{2}\right)$.

We now provide an example, where the conditions of Theorem 1 are not satisfied.

Example 1. Let $E=\mathbf{R}, D=[-9.9,9.9], x_{0}=0$, and define the real function on $D$ by

$$
F(x)=\frac{1}{2400} x^{4}+x-3 .
$$

Using (a)-(c) of Theorem 1 , we can casily obtain, $b_{1}=0, d_{1}=3$, and $a_{1}=.099$. Moreover, condition (d) is not satisfied since it gives $.019<0$, which is not true. Hence, Theorem 1 cannot guarantee that the nethod of tangent parabolas starting from $x_{0}=0$ converges to a solution $x^{*}$ of equation $F(x)=0$ in this case. However we will return back to this example in Remark 2 after our Theorem 2 to show that the method of tangent hyperbolas indeed converges to some $x^{*} \in D$.

Let $a, b, c, d, R$ be given nonnegative constants with $c \in[0,1), x_{0} \in D$ such that $A_{0}^{-1}=A\left(x_{0}\right)^{-1} \in L(E, E), f$ be an increasing real function, which is continuous and nonvanishing on $[0, R]$. Define the polynomial $p$ by

$$
p(t)=\frac{1}{6} a t^{3}+\frac{1}{2} b t^{2}-(1-c) t+d,
$$

the constants $\alpha, \beta$ by

$$
\begin{gathered}
\alpha=\frac{2(1-c)}{b+\sqrt{b^{2}+2 a(1-c)}}, \\
\beta=(1-c) \alpha-\frac{1}{6} a \alpha^{3}-\frac{1}{2} b \alpha^{2},
\end{gathered}
$$


and the iteration $\left\{t_{n}\right\}(n \geq 0)$, by

$$
t_{n+1}=t_{n}-\frac{p\left(t_{n}\right)}{f\left(t_{n}\right)}, \quad t_{0}=0(n \geq 0) .
$$

We need the lemmas:

Lemma 1. The real polynomial $p$ has two positive zeros $r_{1}, r_{2}$ with $r_{1} \leq r_{2}$ and a negative zero $-r_{3}\left(r_{3}>0\right)$ if and only if

$$
d \leq \beta .
$$

Proof. Polynomial $p$ has a negative zero $-r_{3}$, since $p(0)=d>0$, and $p(t)<0$ as $t \rightarrow-\infty$. Moreover, $p^{\prime}(0)=-(1-q)<0$, and $p^{\prime}(t)>0$ as $t \rightarrow+\infty$. Hence, there exists a zero of $p^{\prime}$ in $(0, \infty)$, which by the form of $p$ is given by (4). Thus, $p$ has two positive zeros if and only if

$$
p(\alpha) \leq 0,
$$

which is equivalent to condition (7).

That completes the proof of Lemma 1.

Lemma 2. Assume condition (7) holds, and

$$
f(t) \neq 0, f(t) \leq p^{\prime}(t) \text { for all } t \in\left[0, r_{1}\right] .
$$

Then iteration $\left\{t_{n}\right\}(n \geq 0)$ given by $(10)$ is monotonically increasing and converges to $r_{1}$.

Proof. Define function $g$ by

$$
g(t)=t-\frac{p(t)}{f(t)}
$$

Then by differentiating function $g$ we get

$$
g^{\prime}(t)=\frac{f(t)\left(f(t)-p^{\prime}(t)\right)+f^{\prime}(t) p(t)}{f(t)^{2}} .
$$

It follows from the proof of Lemma $1,(3)$ and (9) that $p^{\prime}(t)<0, p(t)>0$. $f(t)<0$, and $f^{\prime}(t)>0$ for all $t \in\left[0, r_{1}\right]$. Hence, by (11) function $g$ increases on $\left[0, r_{1}\right]$. So, if $t_{k} \in\left[0, r_{1}\right]$ for some $k$, then

$$
t_{k} \leq t_{k}-\frac{p\left(t_{k}\right)}{f\left(t_{k}\right)}=t_{k+1}, \text { and } t_{k+1} \leq t_{k}-\frac{p\left(t_{k}\right)}{f\left(t_{k}\right)} \leq r_{1}-\frac{p\left(r_{1}\right)}{f\left(r_{1}\right)}=r_{1} .
$$


That completes the proof of Lemma 2 .

Remark 1. It can easily be seen by (11) that condition (9) can be replaced by the weaker

$$
f(t) \neq 0, f(t)\left(f(t)-p^{\prime}(t)\right)+f^{\prime}(t) p(t) \geq 0 \text { for all } t \in\left[0, r_{1}\right] .
$$

From now on, we set

$$
A^{-1}(x)=F^{\prime}(x)-\frac{1}{2} F^{\prime \prime}(x) F^{\prime}(x)^{-1} F(x) \quad(x \in D),
$$

and $A\left(x_{n}\right)=A_{n}(n \geq 0)$.

We can now prove the semilocal convergence theorem for the method of tangent hyperbolas (2).

Theorem 2. Let $F: D \subseteq E \rightarrow E$ be a twice Fréchet-differentiable operator. Assume:

(a) there exists $x_{0} \in D$ such that $A_{0}^{-1}=A\left(x_{0}\right)^{-1} \in L(E, E)$;

(b) for all $x \in \bar{U}\left(x_{0}, R\right)$ there exist constants $a, b, c$ such that

$$
\begin{gathered}
\left\|A_{0}^{-1}\left(F^{\prime \prime}(x)-F^{\prime \prime}\left(x_{0}\right)\right)\right\| \leq\left\|x-x_{0}\right\|, \\
\left\|A_{0}^{-1} F^{\prime \prime}\left(x_{0}\right)\right\| \leq b, \\
\left\|A_{0}^{-1}(F \prime(x)-A(x))\right\| \leq c ;
\end{gathered}
$$

(c) conditions (7) and (9) are satisficd for some continuous monotonically increasing and nonvanishing function $f$ on $\left[0, r_{1}\right]$ such that

$$
\left\|A_{0}^{-1}\left(A(x)-A_{0}\right)\right\| \leq f\left(\left\|x-x_{0}\right\|\right)+1<1, \text { for all } x \in \bar{U}\left(x_{0}, r_{1}\right)
$$

and

$$
d \geq\left\|A_{0}^{-1} F\left(x_{0}\right)\right\|
$$

(d) the following hold:

$$
\begin{gathered}
c \in[0,1), \\
r_{2} \leq R, \\
\bar{U}\left(x_{0}, R\right) \subseteq D,
\end{gathered}
$$

where $r_{1}$ and $r_{2}$ are the positive zeros of equation $p(t)=0$, and polynomial $p$ is given by (3). 
Then, the method of tangent hyperbolas $\left\{x_{n}\right\}$ ( $\left.n \geq 0\right)$ generated by (2) is well defined, remains in $\bar{U}\left(x_{0}, r_{1}\right)$ for all $n \geq 0$, and converges to a solution $x^{*} \in \bar{U}\left(x_{0}, r_{1}\right)$ of equation $F(x)=0$. If $r_{1}<r_{2}$ the solution $x^{*}$ is unique in $U\left(x_{0}, r_{2}\right)$, whereas if $r_{1}=r_{2}, x^{*}$ is unique in $\bar{U}\left(x_{0}, r_{1}\right)$.

Moreover, the following error bounds hold for all $n \geq 0$

$$
\left\|x_{n+1}-x_{n}\right\| \leq t_{n+1}-t_{n}
$$

and

$$
\left\|x_{n}-x^{*}\right\| \leq r_{1}-t_{n}
$$

Proof. We first show linear operator $A(x)$ is invertible for all $x \in \bar{U}\left(x_{0}, \alpha\right)$, where $\alpha$ is given by (4). It follows from (15), the Banach lemma on invertible operators [4], [8], the estimate

$$
\left\|A_{0}^{-1}\left(A(x)-A_{0}\right)\right\| \leq f\left(\left\|x-x_{0}\right\|\right)+1<1,
$$

that $A(x)^{-1} \in L(E, E)$, and

$$
\left\|A(x)^{-1} A_{0}\right\| \leq-f\left(\left\|x-x_{0}\right\|\right)^{-1} \leq-f(\alpha)^{-1} .
$$

We must show that estimate (20) holds for all $n \geq 0$. First, note that $x_{1}$ is defined, and by using (2), (6), and (16) we get $\left\|x_{1}-x_{0}\right\|=\left\|-A_{0}^{-1} F\left(x_{0}\right)\right\| \leq$ $d=t_{1}-t_{0}$, which shows (20) for $n=0$. It follows from (22) that linear operator $A\left(x_{1}\right)^{-1} \in L(E, E)$, and hence $x_{2}$ can then be defined by (2). Let $x \in\left[x_{0}, x_{1}\right]=\left\{x: x=\lambda x_{1}+(1-\lambda) x_{0}, 0 \leq \lambda \leq 1\right\}$. By Taylor's formula [5], [7], [10] for a twice Fréchet-differentiable operator $G$ on $D$, we can write

$$
\begin{array}{r}
G(x)=G\left(x_{0}\right)+G^{\prime}\left(x_{0}\right)\left(x-x_{0}\right)+\frac{1}{2} G^{\prime \prime}\left(x_{0}\right)\left(x-x_{0}\right)^{2}+ \\
+\int_{x_{0}}^{x}\left[G^{\prime \prime}(y)-G^{\prime}\left(x_{0}\right)\right](x-y) d y .
\end{array}
$$

Using approximation (23) for $G(x)=A_{0}^{-1} F(x)(x \in D)$, we can get

$$
\begin{gathered}
A_{0}^{-1} F(x)=A_{0}^{-1} F\left(x_{0}\right)+\left(x-x_{0}\right)+A_{0}^{-1}\left(F \prime\left(x_{0}\right)-A_{0}\right)+ \\
+\frac{1}{2} A_{0}^{-1} F^{\prime \prime}\left(x_{0}\right)\left(x-x_{0}\right)^{2}+\int_{x_{0}}^{x} A_{0}^{-1}\left(F^{\prime \prime}(y)-F^{\prime \prime}\left(x_{0}\right)\right)(x-y) d y .
\end{gathered}
$$

Let $\lambda d=s$, then by using (3), (12) - (14), (16), approximation (24) gives

$$
\left\|A_{0}^{-1} F(x)\right\| \leq(1-\lambda) d+c \lambda d+\frac{1}{2} b \lambda^{2} d^{2}+\frac{1}{6} a \lambda^{3} d^{3}=p(s)
$$

(since $\left.x-x_{0}=\lambda\left(x_{1}-x_{0}\right)=-\lambda A_{0}^{-1} F\left(x_{0}\right)\right)$. 
Moreover, by (2), (22) and (25), we get

$$
\left\|x_{2}-x_{1}\right\| \leq\left\|A\left(x_{1}\right)^{-1} A_{0}\right\| \cdot\left\|A_{0}^{-1} F\left(x_{1}\right)\right\| \leq-\frac{p\left(t_{2}\right)}{f\left(t_{1}\right)}=t_{2}-t_{1} .
$$

Similarly, we can show (20) for all $n \geq 0$. Estimate (20) and Lemma 2 imply that the method of tangent hyperbolas $\left\{x_{n}\right\}(n \geq 0)$ is Cauchy in a Banach space $E$, and as such it converges to some $x^{*} \in \bar{U}\left(x_{0}, s\right)$ (since $\bar{U}\left(x_{0}, s\right)$ is a closed set). From (25) and the continuity of $F$ we get $F\left(x^{*}\right)=0$. Furthermore, estimate (21) follows immediately from (20) by using standard majorization techniques [2]. [3]. [4], [5], [6], [8], [10]. To show uniqueness, let $z \in U\left(x_{0}, r_{2}\right)$ with $F(z)=0$. Using (24) for $x=z$, we obtain,

$$
\begin{aligned}
x_{1}-z=A_{0}^{-1}\left(F \prime\left(x_{0}\right)\right. & \left.-A_{0}\right)\left(z-x_{0}\right)+\frac{1}{2} A_{0}^{-1} F^{\prime \prime}\left(x_{0}\right)\left(z-x_{0}\right)^{2} \\
& +\int_{x_{0}}^{z} A_{0}^{-1}\left[F^{\prime \prime}(y)-F^{\prime \prime}\left(x_{0}\right)\right](z-y) d y .
\end{aligned}
$$

We get $\left\|z-x_{0}\right\| \leq r_{1}-t_{0}$ if $z \in \bar{U}\left(x_{0}, r_{1}\right)$, and $\left\|z-x_{0}\right\|=\mu\left(r_{2}-t_{0}\right)$, $0<\mu<1$, if $z \in U\left(x_{0}, r_{2}\right)$. Hence, by (21), and (26), we get for all $n \geq 0:\left\|z-x_{n}\right\| \leq r_{1}-t_{n}$, if $z \in \bar{U}\left(x_{0}, r_{1}\right)$, and $\left\|z-x_{n}\right\| \leq \mu^{n}\left(r_{2}-t_{n}\right)$, if $z \in U\left(x_{0}, r_{2}\right)$. In cither case, we gct $\lim _{n \rightarrow \infty} x_{n}=z$, which yields $x^{*}=z$.

That completes the proof of Theorem 2.

Remark 2. Returning back to Exanıple 1, ancl using (4), (5), (12)-(14), (16), wc get $a=.0495, b=0, c=.0049906, d=3, \alpha=6.1453959$, and $\beta=4.20000143$. Moroever, set

$$
f(t)=\left|F^{\prime}(t)-\frac{1}{2} F^{\prime \prime}(t) F^{\prime}(t)^{-1} F(t)-1\right|-1, \quad t \in\left[0, r_{1}\right] .
$$

It is simple algebra to show that conditions (9) and (15) are satisfied for this choice of $f$. Condition (7) is also satisfied, since $d<\beta$. That is the hypotheses of Theorem 2 are satisfied. Hence, the method of tangent hyperbolas $\left\{x_{n}\right\}$ ( $n \geq 0$ ) given by (2) converges to a solution $x^{*} \in \bar{U}\left(x_{0}, \alpha\right)$ of equation $F(x)=0$. We found $x^{*}=2.967681021$.

Remark 3. Condition (7) can be replaced by a stronger, but easier to check Newton-Kantorovich-type hypothesis [2], [5], [10] as follows: Define polynomials $p_{1}, f_{1}$ by

$$
p_{1}(t)=\frac{1}{2} q_{1} t^{2}-(1-c) t+d, \quad f_{1}(t)=q_{1} t-q_{2},
$$


where

$$
q_{1}=\frac{1}{3} a b+b, \quad \text { and } \quad q_{2}=1-c
$$

It can easily be seen that the conclusions of Theorem 2 hold in the balls $\bar{U}\left(x_{0}, r_{4}\right), U\left(x_{0}, r_{5}\right)$, provided that the Newton-Kantorovich hypothesis

$$
2 q_{1} d \leq(1-c)^{2}
$$

holds, and $r_{4}, r_{5}\left(r_{4} \leq r_{5}\right)$ are the nonnegative zeros of the equation $p_{1}(t)=$ 0 . Note also that since $p(t) \leq p_{1}(t)$ for all $t \in\left[0, r_{2}\right]$, we have $r_{1} \leq r_{4} \leq$ $r_{5} \leq r_{2}$.

Remark 4. Condition (14) can be replaced by

$$
\left\|A_{0}^{-1}\left(F^{\prime}(x)-A(x)\right)\right\| \leq c_{0}+c_{1}\left\|x-x_{0}\right\| \quad \text { for some } c_{0} \geq 0, c_{1} \geq 0
$$

and all $x \in \bar{U}\left(x_{0}, R\right)$. We can also set $c=c_{0}+c_{1} R$. Condition (18) can be replaced by $\alpha \leq R$, but uniqueness is then guaranteed only in $\bar{U}\left(x_{0}, \alpha\right)$.

Remark 5. The results obtained in Theorem 2 can be extended so as to hold a more general setting as follows:

(a) Let $c_{0}, c_{1}$ be nonnegative constants; $v_{1}, v_{2}$ be positive monotonically increasing functions of one variable on $[0, R]$ with $\lim _{t \rightarrow 0} v_{1}(t)=\lim _{t \rightarrow 0} v_{2}(t)=$ 0 such that

$$
\left\|A_{0}^{-1}\left(F^{\prime \prime}(x)-F^{\prime \prime}\left(x_{0}\right)\right)\right\| \leq v_{1}\left(\left\|x-x_{0}\right\|\right)
$$

and

$$
\left\|A_{0}^{-1}\left(F^{\prime}(x)-A(x)\right)\right\| \leq c_{0}+c_{1} v_{2}\left(\left\|x-x_{0}\right\|\right)
$$

for all $x \in U\left(x_{0}, R\right)$.

(b) Function $\bar{p}$ given by

$$
\bar{p}(t)=\int_{0}^{t}(t-r) v_{1}(r) d r+\frac{1}{2} b t^{2}-\left(1-c_{0}-c_{1} v_{2}(t)\right) t+d \quad \text { on }[0, R]
$$

has a unique zero $\varepsilon_{0} \in[0, R]$, and $\bar{p}(R) \leq 0$.

Moreover, set $\varepsilon_{0}=r_{1}$, and $R=r_{2}$. Furthermore, replace conditions (12), (14) by (a), (7) by (b), and polynomial $p$ by function $\bar{p}$ above. Then, under the rest of the hypotheses, as it can easily be seen from the proof, 
the conclusions of Theorem 2 hold in this more general setting. Call such a result Theorem $2^{\prime}$.

Finally, note that for $v_{1}(t)=a t, c_{0}=c$, and $v_{1}(t)=0$ (or $\left.c_{1}=0\right)$, $t \in[0, R]$ function $\bar{p}$ reduces to polynomial $p$ and Theorem $2^{\prime}$ to Theorem 2 .

Finally, we complete this study with the example.

Example 2. We use Theorem 2 to solve quadratic integral equations of the form

$$
x(s)=y(s)+\lambda x(s) \int_{0}^{1} q(s, t) x(t) d t
$$

in the space $E_{1}=C[0,1]$, of all functions continuous on the interval $[0,1]$, with norm

$$
\|x\|=\max _{0 \leq s \leq 1}|x(s)| .
$$

Here we assume that $\lambda$ is a real number called the "albedo" for scattering and the kernel $q(s, t)$ is a continuous function of two variables $s, t$ with $0 \leq s, t \leq 1$ and satisfying

(i) $0<q(s, t)<1,0 \leq s, t \leq 1$ with $q(0,0)=1$;

(ii) $q(s, t)+q(t, s)=1,0 \leq s, t \leq 1$.

The function $y(s)$ is a given continuous function defined on $[0,1]$, and finally $x(s)$ is the unknown function sought in $[0,1]$.

Equations of this type are closely related with the work of S. Chandrasekhar [5], (Nobel Prize of Pliysics 1983), and arise in the theories of radiative transfer, neutron transport and in the kinetic theory of gasses, [4], [5], [10]. There cxists an extensive literature on equations like (27) under various assumptions on the kernel $q(s, t)$ and $\lambda$ is a real or complex number. One can refer to the recent work in [4], [5] and the references there.

For simplicity (without loss of generality) we will assume that

$$
q(s, t)=\frac{s}{s+t} \quad \text { for all } 0 \leq s, t \leq 1 \text { with } q(0,0)=1 .
$$

Note that $q$ so defined satisfies (i) and (ii) above.

Let us now choose $\lambda=.25, y(s)=1$ for all $s \in[0,1]$; and define the operator $H$ on $E_{1}$ by

$$
H(x)=\lambda x(s) \int_{0}^{1} \frac{s}{s+t} x(t) d t-x(s)+1 .
$$

Note that every zero of equation $H(x)=0$ satisfies equation (27). 
Set $x_{0}(s)=1, A_{0}=F^{\prime}\left(x_{0}\right)$, use the definition of the first and second Fréchet-derivatives of the operator $H$ to obtain using Theorem 2,

$$
\begin{gathered}
a=c=0, b=2|\lambda| \max _{0 \leq s \leq 1}\left|\int_{0}^{1} \frac{s}{s+t} d t\right|=2|\lambda| \ln 2=.34657359, f(t)=b t, \\
\bar{\beta}=\left\|H^{\prime}(1)^{-1}\right\|=1.53039421, \\
d \geq\left\|H^{\prime}(1)^{-1} H(t)\right\| \geq \bar{\beta} \lambda \ln 2=.265197107, R=r_{2}=\begin{array}{r}
5.492127839 \text { and } \\
r_{1}=.278652329 .
\end{array}
\end{gathered}
$$

(For detailed computations see also [4], [5]).

The conclusions of Theorem 2 for equation (27) follow since all hypotheses are satisfied. That is there exists a solution $x^{*} \in \bar{U}\left(x_{0}, r_{1}\right)$ of equation (27) which is unique in $U\left(x_{0}, r_{2}\right)$.

\section{References}

[1] M. Altman, Concerning the method of tangent hyperbolas for operator equations, Bull. Acad. Polon. Sci. Ser. Math. Astr. Phys. 9, pp. $633-637,(1961)$.

[2] I.K. Argyros, On the convergence of an Euler-Chebysheff-type method under Newton-Kantorovich-type hypotheses, Pure Mathematics and Applications, 4 3, pp. $369-373$, (1993).

[3] I.K. Argyros, On the method of tangent hyperbolas, J. Approx. Th. Appl. 12 1, pp. $78-96,(1996)$.

[4] I.K. Argyros, Polynomial Operator Equations in Abstract Spaces and Applications, CRC Press LLC, Boca Raton, Florida, (1998).

[5] I.K. Argyros and F. Szidarovszky, The Theory and Applications of Iteration Methods, CRC Press, Boca Raton, Florida, (1993).

[6] J.A. Ezquerro, J.M. Gutierez, and M.A. Hernandez, A construction procedure of iterative methods with cubical convergence, Appl. Math. Comp. (to appear).

[7] L.M. Graves, Riemann integration and Taylor's theorem in general analysis, Trans. Amer. Math. Soc. 29, pp. $163-177$, (1927). 
[8] J.M. Gutierez, A new semilocal convergence theorem for Newton's method, J. Cornput. Appl. Math. 79 (1997), 131-145.

[9] S. Kanno, Convergence theorems for the method of tangent hyperbolas, Math. Japanich, 37, 4, pp. 711-722, (1992).

[10] L.V. Kantorovich and G.P. Akilov, Functional Analysis, Pergamon Press, Oxford, (1982).

[11] R.A. Safiev, The method of tangent hyperbolas, Sov. Math. Dokl. 4, pp. $482-485$, (1963).

Received : March 10, 1998.

\section{Ioannis K. Argyros}

Department of Mathematics

Cameron University

Lawton, OK 73505

U. S. A. 